\title{
Perfect 1-Factorisations of Circulants with Small Degree
}

\author{
Sarada Herke* \\ School of Mathematics and Physics \\ The University of Queensland \\ QLD 4072, Australia \\ sarada.herke@uqconnect.edu. au
}

\author{
Barbara Maenhaut \\ School of Mathematics and Physics \\ The University of Queensland \\ QLD 4072, Australia \\ bmm@maths.uq. edu . au
}

Submitted: Apr 17, 2012; Accepted: Mar 8, 2013; Published: Mar 24, 2013

Mathematics Subject Classifications: 05C70, 05C51

\begin{abstract}
A 1-factorisation of a graph $G$ is a decomposition of $G$ into edge-disjoint 1-factors (perfect matchings), and a perfect 1-factorisation is a 1-factorisation in which the union of any two of the 1-factors is a Hamilton cycle. We consider the problem of the existence of perfect 1-factorisations of even order circulant graphs with small degree. In particular, we characterise the 3-regular circulant graphs that admit a perfect 1-factorisation and we solve the existence problem for a large family of 4-regular circulants. Results of computer searches for perfect 1-factorisations of 4-regular circulant graphs of orders up to 30 are provided and some problems are posed.
\end{abstract}

Keywords: one-factorisation, perfect one-factorisation, circulant graph

\section{Introduction}

A 1-regular spanning subgraph of a graph $G$ is called a 1-factor, or a perfect matching, of $G$. A 1-factorisation of a graph is a partition of its edge set into 1-factors. If a graph $G$ of order $n$ admits a 1-factorisation, then $n$ is even and $G$ is $r$-regular for some positive integer $r$. Thus, in this paper we only consider regular graphs of even order. If the union of every two of the 1-factors of a 1-factorisation is a Hamilton cycle, then the 1factorisation is said to be perfect. We use the abbreviation $\mathrm{P} 1 \mathrm{~F}$ for perfect 1-factorisation. A 1-factorisation $\left\{F_{1}, \ldots, F_{m}\right\}$ is uniform if $F_{i} \cup F_{j}$ is isomorphic to $F_{k} \cup F_{\ell}$ for all $i, j, k, \ell$ where $1 \leqslant i<j \leqslant m$ and $1 \leqslant k<\ell \leqslant m$. We use the abbreviation U1F for uniform 1 -factorisation. Observe that a $\mathrm{P} 1 \mathrm{~F}$ is a $\mathrm{U} 1 \mathrm{~F}$.

\footnotetext{
*Supported by an NSERC PGS D Scholarship and a University of Queensland IPRS and Centennial Scholarship.
} 
The problem of the existence and enumeration of 1-factorisations of complete graphs is a classical problem; see [20] and [26] for a more complete study of the problem. In 1964, Kotzig [14] conjectured that the complete graph $K_{2 n}$ admits a P1F for all $n \geqslant 2$. Perfect 1-factorisations of $K_{2 n}$ have been studied extensively over the past few decades, but Kotzig's conjecture is far from fully resolved. Perfect 1-factorisations of $K_{2 n}$ are known to exist when $n$ is an odd prime [2] and when $2 n-1$ is an odd prime [2, 13] and for many other particular orders (see [22] for a survey of these results and [27] for an up-to-date list of computational results). Perfect 1-factorisations of complete bipartite graphs have also been studied for a number of years; see for example [7, 17]. A graph that admits a P1F has also been called a Hamilton graph [13] and a strongly Hamilton graph $[15,17,28]$.

Cubic graphs that admit a P1F have been studied in $[5,6,12,13,15,19]$. For every even $n \geqslant 4$, there exists a cubic graph of order $n$ that has a P1F (see for example [6]). In [12] Kotzig proved that each cubic graph that admits a P1F can be constructed from the graph consisting of two vertices with three edges between them by repeated application of two modifications. An alternative description of this class of graphs was provided by Bonvicini and Mazzuoccolo [5]. In 1978, Kotzig and Labelle [15] studied particular classes of cubic graphs that admit a P1F and in 1979 [16] they listed a number of open problems about graphs that admit a P1F. Problem 4 in [16] was to show that $G$ admits a P1F if and only if the line graph $L(G)$ admits a P1F. They commented that the problem had been resolved but not published. In 2008, Mazzuoccolo [19] provided a proof of Problem 4 of [16] in the case when $G$ has degree 3. Problem 7 of [16] was to characterise cubic bipartite graphs that admit a P1F, and Problem 9 of [16] was to characterise the 4-regular graphs that admit a $\mathrm{P} 1 \mathrm{~F}$.

The existence of 1-factorisations of circulant graphs was studied in 1980 by Stern and Lenz [24] (see [18] for a survey of the Stern-Lenz theorem). Furthermore, in 1985 Richard Stong [25] showed that every connected Cayley graph on a finite even order abelian group has a 1-factorisation. A graph is said to be Hamiltonian if it contains a Hamilton cycle. There is also a long-standing conjecture that every Cayley graph is Hamiltonian and it is well known that the conjecture is true for Cayley graphs on abelian groups $[9,29]$. In [4] it was shown that a 4-regular connected Cayley graph on a finite abelian group can be decomposed into two Hamilton cycles. However, this result does not guarantee the existence of a P1F.

Here we initiate the study of P1Fs of Cayley graphs by considering the question Which circulant graphs admit a P1F?. We provide a complete answer to this question in the case of 3-regular circulant graphs. We answer this question for 4-regular circulant graphs that are isomorphic to a circulant graph having connection set $\{1,2\}$ or $\{1,3\}$, and observe that having order congruent to $2(\bmod 4)$ is a necessary condition for a circulant graph with connection set $\left\{d_{1}, d_{2}\right\}$ where $d_{1}$ and $d_{2}$ are both odd to have a P1F. The computer results given in the last section of this paper demonstrate however that this necessary condition is not sufficient. This leaves us with the interesting open problem of characterising the 4-regular circulant graphs that admit a P1F. 


\section{Background and Notation}

We only consider simple undirected graphs. We represent the vertex set, edge set, maximum degree and minimum degree of a graph $G$ by $V(G), E(G), \Delta(G)$ and $\delta(G)$, respectively. A graph $G$ is $r$-regular if $\Delta(G)=\delta(G)=r$. We denote a path with $n$ vertices by $P=\left[v_{1}, v_{2}, \ldots, v_{n}\right]$ and a cycle with $n$ vertices by $C=\left(v_{1}, v_{2}, \ldots, v_{n}\right)$. In any graph with vertex set $\mathbb{Z}_{n}$, the edge $\{x, y\}$ is said to have length $k$ where $k$ is the length of the shortest path from $x$ to $y$ in the cycle $(0,1,2, \ldots, n-1)$.

Let $\mathcal{G}$ be a group with identity $e$ and $S \subseteq \mathcal{G}-\{e\}$ such that if $s \in S$ then $s^{-1} \in S$. The Cayley graph $\operatorname{Cay}(\mathcal{G}, S)$ is the graph with vertex set $\mathcal{G}$ and edge set $\{\{g, s g\} \mid g \in$ $\mathcal{G}, s \in S\}$. The set $S$ is called the connection set of Cay $(\mathcal{G}, S)$. A circulant graph is a Cayley graph on a cyclic group. In particular, $\operatorname{Cay}\left(\mathbb{Z}_{n}, R\right)$ will be denoted by $\operatorname{Circ}(n, S)$ where $S=R \cap\left\{1,2, \ldots,\left\lfloor\frac{n}{2}\right\rfloor\right\}$. In this paper, we consider 3-regular and 4-regular circulant graphs, that is, $\operatorname{Circ}\left(n,\left\{d_{1}, d_{2}\right\}\right)$ where $1 \leqslant d_{1}<d_{2} \leqslant \frac{n}{2}$. The graph $\operatorname{Circ}\left(n,\left\{d_{1}, d_{2}\right\}\right)$ is connected if and only if $\operatorname{gcd}\left(d_{1}, d_{2}, n\right)=1$. Every connected graph $\operatorname{Circ}\left(n,\left\{d_{1}, d_{2}\right\}\right)$ is isomorphic to a $\operatorname{graph} \operatorname{Circ}\left(n,\left\{d_{1}^{\prime}, d_{2}^{\prime}\right\}\right)$ for which $\operatorname{gcd}\left(d_{1}^{\prime}, d_{2}^{\prime}\right)=1$ (see for example [3]).

The following definitions are for undirected circulant graphs (there are analogous definitions in the directed case). Let $\mathbb{Z}_{n}^{*}$ denote the set of residues modulo $n$ that are relatively prime to $n$. Observe that $\left|\mathbb{Z}_{n}^{*}\right|=\phi(n)$, where $\phi(n)$ is the Euler-phi function. Two circulant graphs $\operatorname{Circ}\left(n,\left\{d_{1}, d_{2}, \ldots, d_{k}\right\}\right)$ and $\operatorname{Circ}\left(n,\left\{d_{1}^{\prime}, d_{2}^{\prime}, \ldots, d_{k}^{\prime}\right\}\right)$ are said to be conjugate by a multiplier [21] if there exists an $m \in \mathbb{Z}_{n}^{*}$ such that $\left\{m d_{1}^{\prime}, m d_{2}^{\prime}, \ldots, m d_{k}^{\prime}\right\}=$ $\left\{ \pm d_{1}, \pm d_{2}, \ldots, \pm d_{k}\right\}$ modulo $n$. In this case, the permutation $x \mapsto m x$ for $x \in \mathbb{Z}_{n}$ is an isomorphism between $\operatorname{Circ}\left(n,\left\{d_{1}^{\prime}, d_{2}^{\prime}, \ldots, d_{k}^{\prime}\right\}\right)$ and $\operatorname{Circ}\left(n,\left\{d_{1}, d_{2}, \ldots, d_{k}\right\}\right)$. In 1967 Adam conjectured that two circulant graphs are isomorphic if and only if they are conjugate by a multiplier [1]. The conjecture is false in general (see for example [21]). However, in the case where $k=2$ we have the following result $[10,11]$.

Theorem 1. Two 3- or 4-regular circulant graphs $\operatorname{Circ}\left(n,\left\{d_{1}, d_{2}\right\}\right)$ and $\operatorname{Circ}\left(n,\left\{d_{1}^{\prime}, d_{2}^{\prime}\right\}\right)$ are isomorphic if and only if they are conjugate by a multiplier.

Laufer [17] attributes the following theorem to Kotzig [13], however it is stated in Kotzig's paper with different terminology and no proof. Wanless [28] provides a proof in the setting of Latin squares.

Theorem 2. If $G$ is a bipartite $r$-regular graph with $r>2$ and $G$ admits a P1F, then $|V(G)| \equiv 2(\bmod 4)$.

We observe that for even $n$ the circulant graph $G=\operatorname{Circ}\left(n,\left\{d_{1}, d_{2}\right\}\right)$ with $d_{1}, d_{2}$ both odd is bipartite, where the odd and even labeled vertices form the partite sets of $V(G)$. Thus, we obtain the following corollary.

Corollary 3. Suppose $n$ is even and $G=\operatorname{Circ}\left(n,\left\{d_{1}, d_{2}\right\}\right)$, where $d_{1}$ and $d_{2}$ are both odd. If $G$ admits a P1F, then $n \equiv 2(\bmod 4)$. 


\section{$3 \quad$ Degree 3 Circulants}

In this section we characterise the connected 3-regular circulant graphs that admit a P1F. This characterisation is given in Theorem 8. A connected 3-regular circulant graph is a graph of type $\operatorname{Circ}\left(n,\left\{a, \frac{n}{2}\right\}\right)$ with $n \geqslant 4$ even, $1 \leqslant a<\frac{n}{2}$ and $\operatorname{gcd}\left(a, \frac{n}{2}\right)=1$.

Lemma 4. Suppose $n \geqslant 4$ is an even integer. If $a$ is odd and $G=\operatorname{Circ}\left(n,\left\{a, \frac{n}{2}\right\}\right)$ is a connected circulant graph, then $G$ is isomorphic to $\operatorname{Circ}\left(n,\left\{1, \frac{n}{2}\right\}\right)$.

Proof. Since $G$ is connected, $\operatorname{gcd}\left(a, \frac{n}{2}, n\right)=1$. Since $a$ is odd and $n$ is even, if $\operatorname{gcd}(a, n)=$ $d \neq 1$, then $d$ divides $\frac{n}{2}$. Hence $\operatorname{gcd}(a, n)=1$ and by definition, $a \in \mathbb{Z}_{n}^{*}$. Since $a$ is odd, it follows that $a \times \frac{n}{2} \equiv \frac{n}{2}(\bmod n)$, and hence $\left\{a \times 1, a \times \frac{n}{2}\right\}=\left\{a, \frac{n}{2}\right\}(\bmod n)$. Thus $G$ is isomorphic to $\operatorname{Circ}\left(n,\left\{1, \frac{n}{2}\right\}\right)$.

Lemma 5. Suppose $n \geqslant 6$ is an even integer. If $a$ is even and $G=\operatorname{Circ}\left(n,\left\{a, \frac{n}{2}\right\}\right)$ is a connected circulant graph, then $G$ is isomorphic to $\operatorname{Circ}\left(n,\left\{2, \frac{n}{2}\right\}\right)$.

Proof. Since $G$ is connected, $\operatorname{gcd}\left(a, \frac{n}{2}\right)=1$, hence $n \equiv 2(\bmod 4)$, as $a$ is even. We show that $G$ is isomorphic to $\operatorname{Circ}\left(n,\left\{2, \frac{n}{2}\right\}\right)$ by finding an $m \in \mathbb{Z}_{n}^{*}$ such that $\left\{m \times 2, m \times \frac{n}{2}\right\}=$ $\left\{a, \frac{n}{2}\right\}$ modulo $n$.

Case 1: Suppose $a \equiv 0(\bmod 4)$. We claim that $m=\frac{n+a}{2}$ satisfies the above conditions. First, observe that since $n \equiv 2(\bmod 4), m$ is odd. So it is clear that $\left\{m \times 2, m \times \frac{n}{2}\right\}=$ $\left\{n+a, \frac{n}{2}\right\}=\left\{a, \frac{n}{2}\right\}$ modulo $n$. We wish to show that $m \in \mathbb{Z}_{n}^{*}$. Suppose not. Then $\operatorname{gcd}(m, n)=d \neq 1$, so $m=x d, n=y d$ for some integers $x, y$. Hence $a=d(2 x-y)$, so $d$ is a divisor of $a$. Since $m$ is odd and $d$ is a divisor of $m, d$ is odd and therefore $d$ divides $\frac{n}{2}$. Thus $d$ is a common divisor of $a, \frac{n}{2}$ and $n$, which is a contradiction.

Case 2: Suppose $a \equiv 2(\bmod 4)$. We claim that $m=\frac{a}{2}$ satisfies the above conditions. First, observe that $m$ is odd and clearly $\left\{m \times 2, m \times \frac{n}{2}\right\}=\left\{a, \frac{n}{2}\right\}$ modulo $n$. We wish to show that $m \in \mathbb{Z}_{n}^{*}$. Suppose not. Then $\operatorname{gcd}(m, n)=d \neq 1$ and, since $m$ is odd and $d$ is a divisor of $m, d$ is odd and therefore $d$ divides $a$ and $\frac{n}{2}$. Thus $d$ is a common divisor of $a, \frac{n}{2}$ and $n$, which is a contradiction.

Thus, in either case, $G$ is isomorphic to $\operatorname{Circ}\left(n,\left\{2, \frac{n}{2}\right\}\right)$.

Lemma 6. Suppose $n \geqslant 6$ is an even integer. Then $\operatorname{Circ}\left(n,\left\{1, \frac{n}{2}\right\}\right)$ admits a P1F if and only if $n \equiv 2(\bmod 4)$.

Proof. If $n \equiv 2(\bmod 4)$, then define three 1 -factors $A, B, C$ as follows. Let $A$ and $B$ consist of the alternate edges of the cycle formed by the edges of length 1 of $G$, and let $C$ consist of the set of all edges of length $\frac{n}{2}$. It is easy to observe that this is a P1F.

Now let $n \equiv 0(\bmod 4)$, and $\operatorname{suppose}$ that $\operatorname{Circ}\left(n,\left\{1, \frac{n}{2}\right\}\right)$ admits a P1F. Let the edges of the three 1-factors be coloured red, blue and green. By definition, the union of each distinct pair of 1 -factors is a Hamilton cycle. For simplicity, we will call the Hamilton cycle formed by the union of the edges coloured red and green, the red-green cycle, and similarly for the other pairs of colours. It is easy to check that 1-factors $A, B, C$ defined above do not produce a $\mathrm{P} 1 \mathrm{~F}$ when $n \equiv 0(\bmod 4)$ and $n>4$. Thus, not all edges of length $\frac{n}{2}$ can belong to the same 1 -factor. So, without loss of generality, edge $\left\{0, \frac{n}{2}\right\}$ is 
green and edge $\left\{1, \frac{n}{2}+1\right\}$ is red. Since the colours correspond to 1 -factors, edges $\{0,1\}$ and $\left\{\frac{n}{2}, \frac{n}{2}+1\right\}$ are blue and edges $\{1,2\}$ and $\left\{\frac{n}{2}+1, \frac{n}{2}+2\right\}$ are green. Hence $\left\{2, \frac{n}{2}+2\right\}$ must be red or blue. If $\left\{2, \frac{n}{2}+2\right\}$ is red, then there is a red-green 4-cycle, a contradiction, and if $\left\{2, \frac{n}{2}+2\right\}$ is blue, then there is a blue-green 6-cycle, which is again a contradiction. Thus, $\operatorname{Circ}\left(n,\left\{1, \frac{n}{2}\right\}\right)$ does not admit a P1F in the case $n \equiv 0(\bmod 4)$.

We next consider the graph $\operatorname{Circ}\left(n,\left\{2, \frac{n}{2}\right\}\right)$. When $n \equiv 0(\bmod 4), \operatorname{Circ}\left(n,\left\{2, \frac{n}{2}\right\}\right)$ is disconnected, so we consider only the case when $n \equiv 2(\bmod 4)$. It is easy to observe that Circ $(6,\{2,3\})$ admits a P1F. For larger $n$, we have the following lemma.

Lemma 7. Suppose $n>6$ and $n \equiv 2(\bmod 4)$. Then $G=\operatorname{Circ}\left(n,\left\{2, \frac{n}{2}\right\}\right)$ does not admit a P1F.

Proof. Suppose there exists a P1F and let the three 1-factors be coloured red, blue and green.

We first prove that edges $\left\{i, \frac{n}{2}+i\right\}$ and $\left\{i+2, \frac{n}{2}+i+2\right\}$ belong to the same 1-factor for $i=0,1, \ldots, \frac{n}{2}-1$. Suppose this is not the case. Then there exists an $i \in\left\{0,1, \ldots, \frac{n}{2}-1\right\}$ such that $\left\{i, \frac{n}{2}+i\right\}$ is red and $\left\{i+2, \frac{n}{2}+i+2\right\}$ is blue (without loss of generality). Then $\{i, i+2\}$ and $\left\{\frac{n}{2}+i, \frac{n}{2}+i+2\right\}$ are green, and hence $\{i+2, i+4\}$ and $\left\{\frac{n}{2}+i+2, \frac{n}{2}+i+4\right\}$ are red. If $\left\{i+4, \frac{n}{2}+i+4\right\}$ is blue, then there is a 4-cycle in the union of the red and blue 1 -factors, which is a contradiction; if $\left\{i+4, \frac{n}{2}+i+4\right\}$ is green, then there is a 6 -cycle in the union of the red and green 1-factors, a contradiction. Hence the edges $\left\{i, \frac{n}{2}+i\right\}$ and $\left\{i+2, \frac{n}{2}+i+2\right\}$ belong to the same 1 -factor for $i=0,1, \ldots, \frac{n}{2}-1$.

Now observe that the edges of length 2 form two disjoint cycles $C_{1}$ and $C_{2}$ of length $\frac{n}{2}$, which is odd. If all edges of length $\frac{n}{2}$ belong to the same 1-factor, then the edges of $C_{1}$ and $C_{2}$ belong to two 1-factors, which is impossible since they are cycles of odd length. Thus, without loss of generality, we may assume that edge $\left\{0, \frac{n}{2}\right\}$ is red and $\left\{1, \frac{n}{2}+1\right\}$ is blue. It follows that $\left\{x, \frac{n}{2}+x\right\}$ is red for $x=0,2,4, \ldots, \frac{n}{2}-1$ and $\left\{y, \frac{n}{2}+y\right\}$ is blue for $y=1,3,5, \ldots, \frac{n}{2}-2$. Thus, $\left\{\frac{n}{2}-2, n-2\right\}$ is blue and $\left\{\frac{n}{2}, 0\right\}$ is red, which is a contradiction.

Therefore $G$ does not admit a P1F.

The preceeding four lemmas are now used to prove the following characterisation result.

Theorem 8. Let $n \geqslant 4$ be an even integer and let $a$ be an integer in the range $1 \leqslant a<\frac{n}{2}$. Then $\operatorname{Circ}\left(n,\left\{a, \frac{n}{2}\right\}\right)$ has a P1F if and only if one of the following cases occurs:

- $n=4$ and $a=1$;

- $n=6$ and $a=1$ or $a=2$;

- $n>6, n \equiv 2(\bmod 4)$, $a$ is odd and $\operatorname{gcd}\left(a, \frac{n}{2}\right)=1$.

Proof. Observe that the construction of a P1F for $\operatorname{Circ}(4,\{1,2\})$ and $\operatorname{Circ}(6,\{a, 3\})$, $a=1,2$ is trivial. The condition $\operatorname{gcd}\left(a, \frac{n}{2}\right)=1$ is needed in order for $\operatorname{Circ}\left(n,\left\{a, \frac{n}{2}\right\}\right)$ to be 
connected. If $a$ is odd, then by Lemma $4, G$ is isomorphic to $\operatorname{Circ}\left(n,\left\{1, \frac{n}{2}\right\}\right)$, which, by Lemma 6 has a P1F if and only if $n \equiv 2(\bmod 4)$. If $a$ is even, then by Lemma $5, G$ is isomorphic to $\operatorname{Circ}\left(n,\left\{2, \frac{n}{2}\right\}\right)$, which, by Lemma 7 , does not admit a P1F.

\section{Degree 4 Circulants}

In this section, we consider the 4-regular circulant graphs with connection sets $\{1,3\}$ and $\{1,2\}$. We prove the existence and non-existence of P1Fs for circulant graphs with these two connection sets, respectively. We note that $\operatorname{Circ}(n,\{1,3\})$ is bipartite, hence, by Corollary 3 , it admits a P1F only if $n \equiv 2(\bmod 4)$.

Theorem 9. If $n \equiv 2(\bmod 4)$ and $n>6$, then $\operatorname{Circ}(n,\{1,3\})$ admits a P1F.

Proof. Let $G=\operatorname{Circ}(n,\{1,3\})$. For $t=5,6,7,8$ and $n \geqslant 10$ define

$$
E_{t}=\left\{\{x, x+3\},\{x+1, x+2\} \mid x=4 k+t, k \in\left\{0,1, \ldots, \frac{n-10}{4}\right\}\right\},
$$

where $x+i$ is calculated modulo $n$. We construct four disjoint 1 -factors $A, B, C, D$ as follows (see Figure 1).

$$
\begin{aligned}
& A=\{\{0,1\},\{2,3\},\{4,5\}\} \cup E_{6} \\
& B=\{\{1,2\},\{3,4\},\{5,6\}\} \cup E_{7} ; \\
& C=\{\{n-1,2\},\{0,3\},\{1,4\}\} \cup E_{5} ; \\
& D=\{\{2,5\},\{3,6\},\{4,7\}\} \cup E_{8} .
\end{aligned}
$$

Notice that, in each 1-factor, the edges form a pattern that repeats every 4 vertices, with the exception of three edges on 6 initial vertices. Since $n-6 \equiv 0(\bmod 4)$, the pattern fits into the remaining $n-6$ vertices. The 1 -factors $A, B, C, D$ clearly form a 1 -factorisation of $G$. We now check the six possible unions of these 1-factors.

1) $\underline{A}$ and $B$ :

The union of the 1-factors $A$ and $B$ is the union of the paths $P=[0,1,2,3,4,5,6]$ and $Q_{k}$ for $k=0,1, \ldots, \frac{n-10}{4}$, where $Q_{k}=[6+4 k, 9+4 k, 8+4 k, 7+4 k, 10+4 k]$ and all vertex labels are computed modulo $n$. This forms a Hamilton cycle.

2) $A$ and $C$ :

The union of the 1 -factors $A$ and $C$ is the union of the paths $P=[n-1,2,3,0,1,4,5]$ and $Q_{k}$ for $k=0,1, \ldots, \frac{n-10}{4}$, where $Q_{k}=[5+4 k, 8+4 k, 7+4 k, 6+4 k, 9+4 k]$ and all vertex labels are computed modulo $n$. This forms a Hamilton cycle.

3) $A$ and $D$ :

If $n=10$, then the union of the 1 -factors $A$ and $D$ is the cycle $(7,4,5,2,3,6,9,0,1,8)$, which is a Hamilton cycle. If $n>10$ then the union of the 1 -factors $A$ and $D$ is the union of the paths $P_{1}=[7,4,5,2,3,6], P_{2}=[0,1], Q_{k}$ and $R_{k}$ for $k=0,1, \ldots, \frac{n-10}{4}$, where 


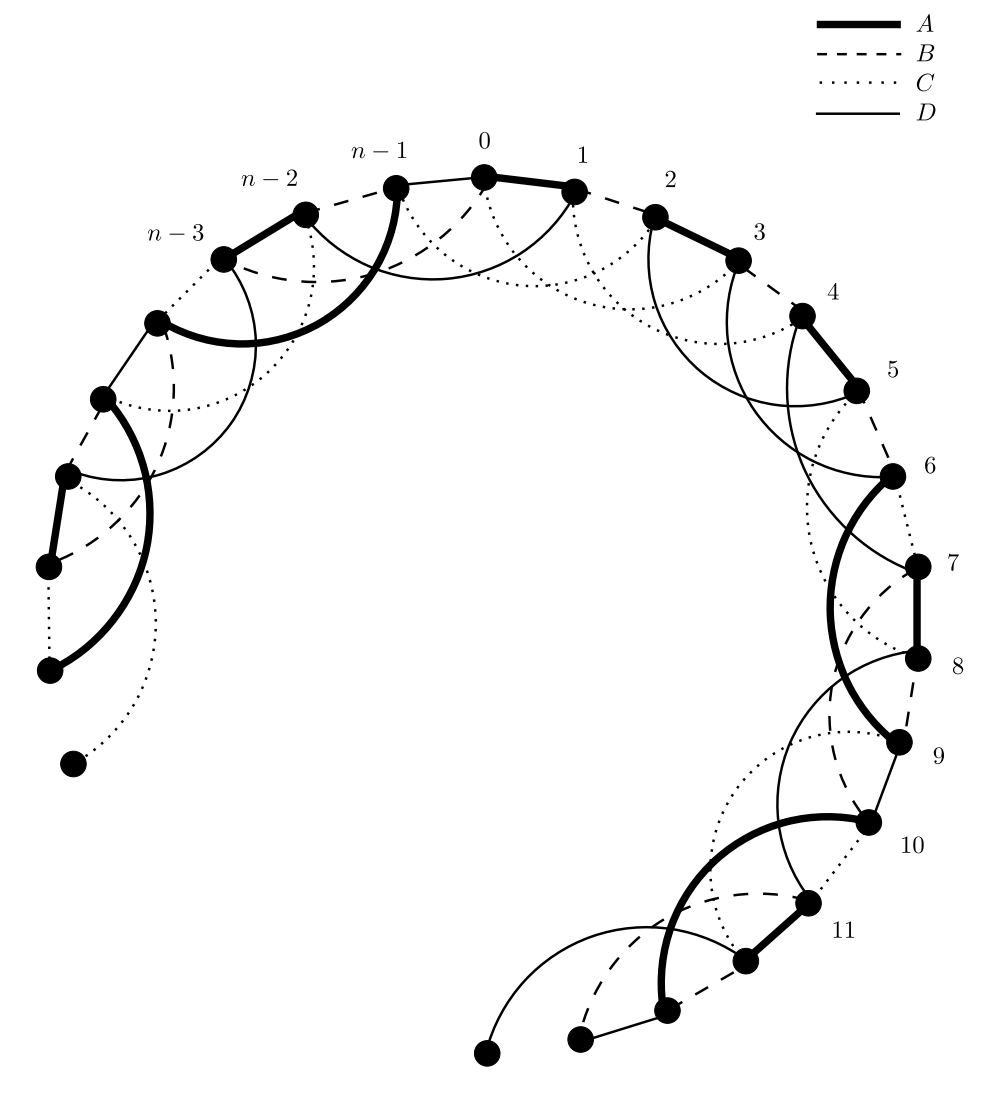

Figure 1: A P1F of $\operatorname{Circ}(n,\{1,3\})$ when $n \equiv 2(\bmod 4)$.

$Q_{k}=[6+4 k, 9+4 k, 10+4 k]$ and $R_{k}=[7+4 k, 8+4 k, 11+4 k]$ and all vertex labels are computed modulo $n$. This forms a Hamilton cycle.

4) $B$ and $C$ :

If $n=10$, then the union of the edges in $B$ and $C$ is the cycle $(0,3,4,1,2,9,8,5,6,7)$, which is a Hamilton cycle. If $n>10$ then the union of the edges in $B$ and $C$ is the union of the paths $P_{1}=[n-1,2,1,4,3,0], P_{2}=[5,6], Q_{k}$ and $R_{k}$ for $k=0,1, \ldots, \frac{n-10}{4}$, where $Q_{k}=[5+4 k, 8+4 k, 9+4 k]$ and $R_{k}=[6+4 k, 7+4 k, 10+4 k]$ and all vertex labels are computed modulo $n$. This forms a Hamilton cycle.

5) $B$ and $D$ :

The union of the 1 -factors $B$ and $D$ is the union of the paths $P=[1,2,5,6,3,4,7]$ and $Q_{k}$ for $k=0,1, \ldots, \frac{n-10}{4}$, where $Q_{k}=[7+4 k, 10+4 k, 9+4 k, 8+4 k, 11+4 k]$ and all vertex labels are computed modulo $n$. This forms a Hamilton cycle.

6) $C$ and $D$ :

If $n=10$, then the union of the 1 -factors $C$ and $D$ is the cycle $(1,4,7,6,3,0,9,2,5,8)$, which is a Hamilton cycle. If $n>10$, then the union of the 1 -factors $C$ and $D$ is the union of the paths $P=[n-2,1,4,7,6,3,0, n-1,2,5,8]$ and $Q_{k}$ for $k=0,1, \ldots, \frac{n-14}{4}$, 
where $Q_{k}=[8+4 k, 11+4 k, 10+4 k, 9+4 k, 12+4 k]$ and all vertex labels are computed modulo $n$. This forms a Hamilton cycle.

Since each pair of 1 -factors forms a Hamilton cycle, we have constructed a P1F of $G$.

Lemma 10. Suppose $n$ is even and $n>10$. There are exactly $\frac{\phi(n)}{2}$ circulant graphs of the form $\operatorname{Circ}\left(n,\left\{d_{1}, d_{2}\right\}\right)$ with $1 \leqslant d_{1}<d_{2}<\frac{n}{2}$ that are isomorphic to $\operatorname{Circ}(n,\{1,3\})$.

Proof. Suppose $n$ is even and $n>10$ and let $G=\operatorname{Circ}(n,\{1,3\})$. Since $d_{1}, d_{2}<\frac{n}{2}$, by Theorem 1, the number of graphs of the form $\operatorname{Circ}\left(n,\left\{d_{1}, d_{2}\right\}\right)$ that are isomorphic to $G$ is at most $\frac{\phi(n)}{2}$. Suppose there are fewer than $\frac{\phi(n)}{2}$ such graphs. Then there are two distinct integers $m$ and $m^{\prime}$ belonging to the first half of the residues in $\mathbb{Z}_{n}^{*}$ such that $m \neq \pm m^{\prime}$, and $\{m, 3 m\}=\left\{ \pm m^{\prime}, \pm 3 m^{\prime}\right\}(\bmod n)$. Then $m= \pm 3 m^{\prime}$ and $3 m= \pm m^{\prime}$, so $9 m^{\prime}= \pm m^{\prime}$, and since $m^{\prime}$ is nonzero, this implies that $9= \pm 1(\bmod n)$. Hence $n=8$ or 10 , a contradiction, and thus the result follows.

Since Lemma 10 applies for $n>10$, we briefly mention the smaller cases here. When $n=6$ the only 4-regular circulant graph is $\operatorname{Circ}(6,\{1,2\})$ and it is trivial to check that it admits a P1F. When $n=10$, Circ $(10,\{1,3\})$, admits a P1F by Theorem 9. It is not difficult to show that any other 4-regular circulant graph of the form $\operatorname{Circ}\left(10,\left\{d_{1}, d_{2}\right\}\right)$ does not admit a P1F (see also the computer search results in Section 5).

The next proof requires a new definition, similar to one given in [8]. Consider the following set of edges of $\operatorname{Circ}\left(n,\left\{d_{1}, d_{2}, \ldots, d_{k}\right\}\right)$ :

$$
E=\left\{\left\{-d_{i}, 0\right\},\left\{-d_{i}+1,1\right\}, \ldots,\left\{-1, d_{i}-1\right\} \mid i=1,2, \ldots, k\right\} .
$$

We observe that $|E|=d_{1}+d_{2}+\cdots+d_{k}$. Informally, $E$ is the set of edges crossing the dotted line segment $L$ between vertices 0 and $n-1$ (see Figure 2). We can similarly define a set of edges crossing a dotted line segment between vertices 0 and 1 , or 1 and 2 , etc. We call such a dotted line segment $L$ a crossing line and we call the associated set of edges $E$ the crossing set of $L$.

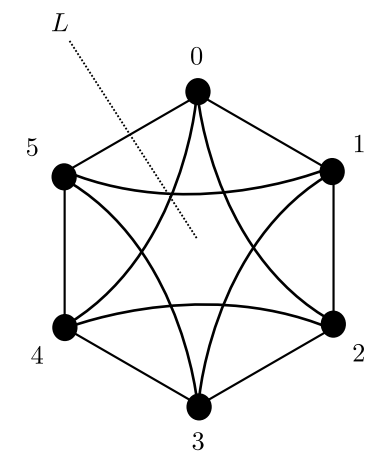

Figure 2: A crossing line $L$ between 5 and 0 in $\operatorname{Circ}(6,\{1,2\})$ with crossing set $\{\{0,5\},\{0,4\},\{1,5\}\}$. 
Theorem 11. Suppose $n>2$ is even. The graph $\operatorname{Circ}(n,\{1,2\})$ admits a P1F if and only if $n \in\{4,6\}$.

Proof. It is clear that $\operatorname{Circ}(4,\{1,2\})$ and $\operatorname{Circ}(6,\{1,2\})$ each have a P1F. Suppose that $G=\operatorname{Circ}(n,\{1,2\})$ admits a $\mathrm{P} 1 \mathrm{~F}$ and assume $n>6$. Hence $G$ is 4 -regular. Let the edges of the four 1-factors be coloured red, blue, green and yellow. By our assumption, the union of each distinct pair of 1-factors is a Hamilton cycle. For simplicity, we will call the Hamilton cycle formed by the union of the edges coloured red and green, the red-green cycle, and similarly for the other pairs of colours.

Consider a crossing line $L$ with crossing set $E$. We say that $L$ has a $k$-configuration if $k$ distinct colours are used on the edges of $E$. Clearly $|E|=3$ and these edges cannot all be the same colour, since the colouring arose from a 1-factorisation. Thus $L$ must have either a 2-configuration or a 3-configuration. Notice that if $L$ has a 2-configuration, the two edges of $E$ with the same colour must be the edges of length 2 .

We first show that there exists a crossing line with a 3-configuration. Suppose there does not. Let $L$ be any crossing line, which necessarily has a 2-configuration and without loss of generality, say the colours are red and green, where red is the colour of the edge of length 1 . Without loss of generality, let the red edge have end-vertices labeled 1 and 2 , so that the green edges are $\{0,2\}$ and $\{1,3\}$ (see Figure 3). Now the edges $\{2,3\}$ and $\{2,4\}$ must be coloured blue and yellow, and then the crossing line $L^{\prime}$ that has $\{2,3\}$ in its crossing set has a 3 -configuration, which is a contradiction.

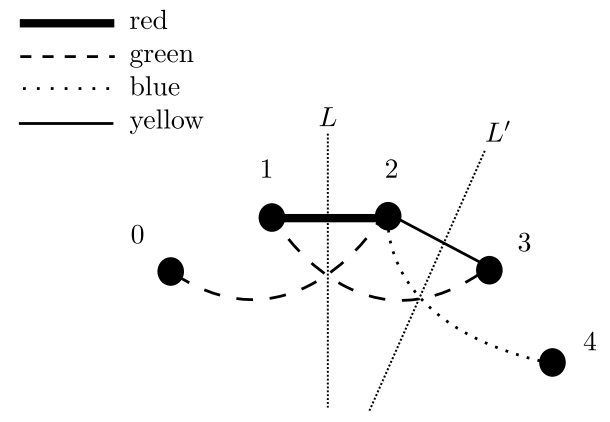

Figure 3: A 3-configuration must occur.

Now take $L$ to be a crossing line in $G$ with a 3 -configuration. Without loss of generality, we assume that the corresponding crossing set is $E=\{\{1,2\},\{0,2\},\{1,3\}\}$ with $\{1,2\}$ coloured red, $\{0,2\}$ coloured blue and $\{1,3\}$ coloured green. It follows that $\{0,1\}$ and $\{2,3\}$ are coloured yellow, $\{1, n-1\}$ is coloured blue, and $\{2,4\}$ is coloured green, see Figure 4 .

The edge $\{0, n-1\}$ must be coloured red or green. If it is red, then $(0, n-1,1,2)$ is a red-blue 4-cycle, which is not Hamiltonian as $n>4$. Hence $\{0, n-1\}$ is green. The edge $\{3,4\}$ must be coloured red or blue. If it is red, then $(1,2,4,3)$ is a red-green 4-cycle, which is not Hamiltonian. Thus $\{3,4\}$ is blue and we find a blue-green 6 -cycle $(0, n-1,1,3,4,2)$, which is not Hamiltonian as $n>6$. Therefore, it is proved that $\operatorname{Circ}(n,\{1,2\})$ with $n>6$ does not admit a P1F. 


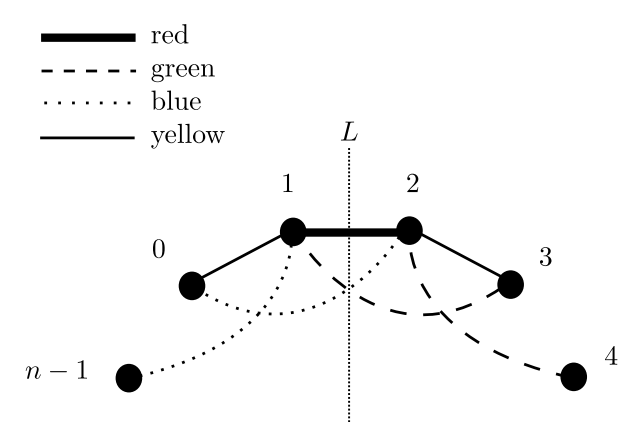

Figure 4: This configuration must occur in $\operatorname{Circ}(n,\{1,2\})$.

It is clear that for $n=4,6$, the only possible uniform 1 -factorisations of $\operatorname{Circ}(n,\{1,2\})$ are perfect 1-factorisations. For $n>6$, we prove the following theorem.

Theorem 12. Suppose $n$ is an even integer and $n>6$. Then $\operatorname{Circ}(n,\{1,2\})$ does not admit a uniform 1-factorisation.

Proof. Suppose that $G=\operatorname{Circ}(n,\{1,2\})$ admits a uniform 1-factorisation of some type. Since $n>6, G$ is 4-regular. Let the edges of the four 1-factors in the uniform 1factorisation be coloured red, blue, green and yellow. By definition, the graphs with edge sets given by the union of each distinct pair of 1-factors are all isomorphic. For simplicity, we will call the graph formed by the red and green edges, the red-green graph, and similarly for the other pairs of colours.

Consider a crossing line $L$ with crossing set $E$. As in the proof of Theorem 11, there exists a crossing line with a 3-configuration, and without loss of generality, we assume that the corresponding crossing set is $E=\{\{1,2\},\{0,2\},\{1,3\}\}$ with $\{1,2\}$ coloured red, $\{0,2\}$ coloured blue and $\{1,3\}$ coloured green. Since each colour corresponds to a 1 -factor, it follows that edges $\{0,1\}$ and $\{2,3\}$ are yellow and edge $\{n-1,1\}$ is blue, as in Figure 4.

We claim that the yellow-blue graph is a Hamilton cycle. Suppose it is not. Let $C=\left(n-1,1,0,2,3, c_{1}, c_{2}, \ldots, c_{t}\right)$ be the cycle in the yellow-blue graph that crosses the crossing line $L$. Since $C$ is not a Hamilton cycle, it follows that there is a set of vertices $A=\left\{a_{1}, a_{2}, \ldots, a_{k}\right\}$ that are not in the vertex set of $C$. Note that $A \subseteq\{4,5, \ldots, n-2\}$. Considering the path $\left[3, c_{1}, c_{2} \ldots, c_{t}, n-1\right]$ in the cycle $C$, it is clear that the set $A$ cannot contain a pair of consecutive vertices. Using only edges of length 2 , it is impossible to create a cycle on any subset of the vertices in $A$, and so the yellow-blue graph cannot contain a second cycle. Thus $C$ must be a Hamilton cycle, and since yellow and blue were 1-factors of a uniform 1-factorisation, it follows that the 1-factorisation is perfect. This contradicts Theorem 11 and hence the result is proved. 


\section{Computer Searches and Conjectures}

We ran computer searches using orderly algorithms based on the ideas in [23] to address the question of the existence of perfect 1-factorisations of 4-regular circulant graphs. We also used Theorem 1 to determine which of these circulant graphs are isomorphic. Table 1 summarises the results of the computer searches for P1F's in circulant graphs of type $\operatorname{Circ}\left(n,\left\{d_{1}, d_{2}\right\}\right)$ where $1 \leqslant d_{1}<d_{2}<\frac{n}{2}$ with small order $(n \leqslant 30)$. The table provides one connection set for each relevant isomorphism class.

\begin{tabular}{|c|c|c|}
\hline$n$ & $\left\{d_{1}, d_{2}\right\}$ for which a P1F exists & $\left\{d_{1}, d_{2}\right\}$ for which a P1F does not exist \\
\hline 6 & $\{1,2\}$ & none \\
\hline 8 & none & all \\
\hline 10 & $\{1,3\}$ & $\{1,2\},\{1,4\}$ \\
\hline 12 & none & all \\
\hline 14 & $\{1,3\}$ & $\{1,2\},\{1,4\},\{1,6\}$ \\
\hline 16 & none & all \\
\hline 18 & $\{1,3\},\{1,5\}$ & $\{1,2\},\{1,4\},\{1,6\},\{1,8\},\{2,3\}$ \\
\hline 20 & none & all \\
\hline 22 & $\{1,3\},\{1,5\}$ & all \\
\hline 24 & none & $\{1,2\},\{1,4\},\{1,6\},\{1,8\},\{1,10\},\{1,12\}$ \\
\hline 26 & $\{1,3\},\{1,5\},\{1,7\}$ & all \\
\hline 28 & none & $\{1,2\},\{1,4\},\{1,6\},\{1,8\},\{1,10\},\{1,11\}$, \\
\hline 30 & $\{1,3\},\{1,5\},\{1,7\},\{1,9\},\{3,5\}$ & $\{1,12\},\{1,14\},\{2,3\},\{2,5\},\{2,9\},\{5,6\}$ \\
\hline
\end{tabular}

Table 1: P1Fs of 4-regular circulants

Since $n$ is even, if $d_{1}$ and $d_{2}$ are both even, then $\operatorname{Circ}\left(n,\left\{d_{1}, d_{2}\right\}\right)$ is disconnected. Thus, the two cases to consider are when exactly one of $d_{1}$ and $d_{2}$ is odd and when they are both odd. Recall that for even $n$, a circulant $\operatorname{Circ}\left(n,\left\{d_{1}, d_{2}\right\}\right)$ is bipartite if and only if $d_{1}$ and $d_{2}$ are both odd. By Corollary 3, a bipartite circulant $\operatorname{Circ}\left(n,\left\{d_{1}, d_{2}\right\}\right)$ with $n \equiv 0(\bmod 4)$ does not admit a P1F. The computer results indidicate that all 4-regular bipartite circulants of order $n \equiv 2(\bmod 4)$ have a $\mathrm{P} 1 \mathrm{~F}$ for $8 \leqslant n \leqslant 28$. This is not true in general, however, since further computer searching showed that $\operatorname{Circ}(30,\{1,11\})$ does not have a P1F. Thus, we pose the following problem.

Problem 13. Characterise the 4-regular bipartite circulant graphs of order $2(\bmod 4)$ that admit a P1F.

If $\operatorname{Circ}\left(n,\left\{d_{1}, d_{2}\right\}\right)$ is a 4-regular circulant and exactly one of $d_{1}$ and $d_{2}$ is odd, the computer search indicates that $G$ does not have a P1F for $n \leqslant 30$. Based on these results, as well as the non-existence result in Theorem 11, we make the following conjecture.

Conjecture 14. Suppose $n$ is even and $n>6$. If $G=\operatorname{Circ}\left(n,\left\{d_{1}, d_{2}\right\}\right)$ is a 4-regular circulant graph and one of $d_{1}, d_{2}$ is odd and the other is even, then $G$ does not admit a $\mathrm{P} 1 \mathrm{~F}$. 


\section{References}

[1] A. Adam. Research problems 2-10. J. Combin. Theory, 2: 393, 1967.

[2] B.A. Anderson. Finite topologies and Hamiltonian paths. J. Combin. Theory Ser. B, 14: 87-93, 1973.

[3] J. Barajas and O. Serra. On the chromatic number of circulants. Discrete Math., 309: 5687-5696, 2009.

[4] J.C. Bermond, O. Favaron, and M. Maheo. Hamiltonian decomposition of Cayley graphs of degree 4. J. Combin. Theory Ser. B, 46: 142-153, 1989.

[5] S. Bonvicini and G. Mazzuoccolo. A new description of perfectly one-factorable cubic graphs. Atti Semin. Mat. Fis. Univ. Modena Reggio Emilia, 54: 167-173, 2006.

[6] S. Bonvicini and G. Mazzuoccolo. Perfect one-factorizations in generalized Petersen graphs. Ars Combin., 99: 33-43, 2011.

[7] D. Bryant, B.M. Maenhaut, and I.M. Wanless. A family of perfect factorisations of complete bipartite graphs. J. Combin. Theory Ser. A, 98: 328-342, 2002.

[8] D. Bryant and G. Martin. Some results on decompositions of low degree circulant graphs. Australas. J. Combin., 45: 251-261, 2009.

[9] S.J. Curran and J.A. Gallian. Hamiltonian cycles and paths in Cayley graphs and digraphs - a survey. Discrete Math., 156: 1-18, 1996.

[10] C. Delorme, O. Favaron, and M. Maheo. Isomorphisms of Cayley multigraphs of degree 4 on finite ablelian groups. European J. Combin., 13: 59-61, 1992.

[11] X. Fang and M. Xu. On isomorphisms of Cayley graphs of small valency. Algebra Colloq., 1: 67-76, 1994.

[12] A. Kotzig. Construction of third-order Hamiltonian graphs. Časop. Pěst. Mat., 87: 148-168, 1962.

[13] A. Kotzig. Hamilton graphs and Hamilton circuits. Theory of Graphs and its Applications (Proc. Sympos. Smolenice 1963) Nakl. CSAV, Praha, 63-82, 1964.

[14] A. Kotzig. Problem 20. Theory of Graphs and its Applications (Proc. Sympos. Smolenice 1963) Nakl. CSAV, Praha 162, 1964.

[15] A. Kotzig and J. Labelle. Strongly Hamiltonian graphs. Utilitas. Math., 14: 99-116, 1978.

[16] A. Kotzig and J. Labelle. Quelques problèmes ouverts concernant les graphes fortement hamiltoniens. Ann. Sci. Math. Québec, 3: 95-106, 1979.

[17] P.J. Laufer. On strongly Hamilton complete bipartite graphs. Ars. Combin., 9: 43-46, 1980.

[18] C.C. Lindner and A.P. Street. The Stern-Lenz Theorem: background and applications. Congr. Numer., 80: 5-21, 1991.

[19] G. Mazzuoccolo. Perfect one-factorizations in line-graphs and planar graphs. Australas. J. Combin., 41: 227-233, 2008. 
[20] E. Mendelsohn and A. Rosa. One-factorizations of the complete graph - a survey. J. Graph Theory, 9: 43-65, 1985.

[21] M. Muzychuk. A solution of the isomorphism problem for circulant graphs. Proc. London. Math. Soc., 88: 1-41, 2004.

[22] E. Seah. Perfect one-factorizations of the complete graph - a survey. Bull. Inst. Combin. App., 1: 59-70, 1991.

[23] E. Seah and D.R. Stinson. Some perfect one-factorizations of $K_{14}$. Ann. Discrete Math., 34: 419-436, 1987.

[24] G. Stern and H. Lenz. Steiner triple systems with given subspaces: another proof of the Doyen-Wilson theorem. Boll. Un. Mat. Ital. Ser. A, 17: 109-114, 1980.

[25] R. Stong. On 1-factorizability of Cayley graphs. J. Combin. Theory Ser. B, 39: 298-307, 1985.

[26] W. D. Wallis. One-factorizations. in Math. Appl., Volume 390, Kluwer Academic, Dordrecht, 1997.

[27] I.M. Wanless. New perfect 1-factorisations. http://users.monash. edu . au/ iwanless/data/P1F/newP1F.html

[28] I.M. Wanless. Perfect factorisations of bipartite graphs and Latin squares without proper subrectangles. Electron. J. Combin., 6: R9, 1999.

[29] D. Witte and J.A. Gallian. A survey: Hamiltonian cycles in Cayley graphs. Discrete Math., 51: 293-304, 1984. 